\title{
Complexidade taxonômica e ampliação da área de ocorrência de Attalea phalerata Mart. ex Spreng. para o Estado do Amapá, Brasil
}

Taxonomic complexity and occurrence area expansion of the Attalea phalerata Mart. ex Spreng. to the State of Amapá, Brazil

Complejidad taxonômica y ampliación del área de incidente de Attalea phalerata Mart. ex Spreng. para el Estado de Amapá, Brasil

Tonny David Santiago Medeiros ORCID: https://orcid.org/0000-0002-0891-1584 Instituto de Pesquisas Científicas e Tecnológicas do Estado do Amapá, Brasil E-mail: tonnyiepa@gmail.com

Ana Cláudia Lira Guedes ORCID: https://orcid.org/0000-0001-9200-4727 Empresa Brasileira de Pesquisa Agropecuária -Amapá, Brasil E-mail: ana-lira.guedes@embrapa.br

Carlos Alberto Santos da Silva Júnior ORCID: https://orcid.org/0000-0002-2329-7754 Instituto de Pesquisas Científicas e Tecnológicas do Estado do Amapá, Brasil E-mail: garffyld29@gmail.com

Raullyan Borja Lima e Silva ORCID: https://orcid.org/0000-0003-4104-5611 Instituto de Pesquisas Científicas e Tecnológicas do Estado do Amapá, Brasil E-mail: raullyanborja@uol.com.br

Ana Luzia Ferreira Farias

ORCID: https://orcid.org/0000-0002-5338-6598 Universidade do Estado do Amapá Brasil E-mail: analuziafarias@gmail.com

Sheylla Susan Moreira da Silva de Almeida ORCID: https://orcid.org/0000-0002-7687-8288 Universidade Federal do Amapá, Brasil E-mail: sheyllasusan@yahoo.com.br

Adelson Rocha Dantas

ORCID: https://orcid.org/0000-0001-6213-5953 Instituto Nacional de Pesquisas da Amazônia, Brasil

E-mail: adelson.dantas@yahoo.com.br

Salustiano Vilar da Costa Neto

ORCID: https://orcid.org/0000-0002-1459-3658 Instituto de Pesquisas Científicas e Tecnológicas do Estado do Amapá, Brasil E-mail: salucostaneto@gmail.com

Patrick de Castro Cantuária

ORCID: https://orcid.org/0000-0002-3676-7866 Instituto de Pesquisas Científicas e Tecnológicas do Estado do Amapá, Brasil E-mail: patrickcantuaria@gmail.com

\begin{abstract}
Resumo
O objetivo desta pesquisa é discutir a complexidade taxonômica e a ampliação da área de ocorrência de Attalea phalerata, Amapá, Brasil. A coleta do material foi realizada no município de Mazagão, área experimental da EMBRAPA, Amapá. A descrição e a morfometria foram realizadas no Herbário Amapaense (HAMAB), do Instituto de Pesquisas Científicas e Tecnológicas do Estado do Amapá (IEPA). A prancha foi composta utilizando-se o software Adobe Photoshop e a análise morfométrica foi realizada in vivo. Os resultados indicam diversos sinônimos atribuídos à espécie, por consequência do rearranjo na definição das espécies do gênero Attalea. Os resultados deste estudo possibilitam ao conhecimento da flora brasileira ampliar a distribuição geográfica A. phalerata para o Estado do Amapá. Conclui-se que a importância da taxonomia vegetal se justifica, dentre vários aspectos, no sentido de esclarecer e sustentar o emprego das denominações específicas e genéricas aceitas, com base no atual Sistema de Classificação, mas também, reforça a importância da Taxonomia Clássica para a Ciência Moderna.

Palavras-chave: Amazônia; Arecaceae; Distribuição geográfica; Urucuri.
\end{abstract}




\begin{abstract}
The objective of this research is to discution how synonyms and the expansion of the occurrence area in Brazil of Attalea phalerata, in the region of Amazon River, State of Amapá. The material was collected in the municipality of Mazagão, an experimental area of EMBRAPA, Amapá. The description and morphometry were carried out at Amapaense Herbarium (HAMAB), of the Instituto de Pesquisas Científicas e Tecnológicas do Estado do Amapá (IEPA). The board was composed using Adobe Photoshop software and the morphometric analysis was performed in vivo. The results indicate several synonyms attributed to the species, as a result of the rearrangement in the definition of the species of the genus Attalea. The results of this study enable knowledge of Brazilian flora to expand the geographical distribution of A. phalerata to the State of Amapá. It is concluded that the importance of plant taxonomy is justified, among several aspects, in order to clarify and support the use of specific and generic denominations accepted, based on the current Classification System, but also, it reinforces the importance of Classical Taxonomy for the Modern Science.
\end{abstract}

Keywords: Amazon; Arecaceae; Geographic distribution; Urucuri.

\title{
Resumen
}

El objetivo de esta pregunta es hablar de la complejidad taxonômica y la ampliación del área de incidente de Attalea phalerata, Amapá, Brasil. La colección del material se realizó en la autoridad local de Mazagão, el área experimental del EMBRAPA, Amapá. La descripción y el morfometria se realizaron en el Herbarium de Amapá (HAMAB), del Instituto de Pesquisas Científicas e Tecnológicas do Estado de Amapá (IEPA). El tablón se formó cuando el Adobe Photoshop se usa el software y el análisis morfométrica se realizaron en la criatura viva. Los resultados indican varios sinónimos atribuidos a la clase, por consiguiente, del cambio de lugar en la definición de las clases del tipo Attalea. Los resultados de este estudio hacen posible al conocimiento de la flora brasileña amplía la distribución geográfica de A. phalerata para el estado de Amapá. Terminar lo que la importancia de la taxonomía de verduras justifica, entre varios aspectos, en dirección de explicación y apoyo del trabajo de las denominaciones específicas y genéricas aceptadas, en la base del sistema actual de la Clasificación, sino también, refuerza la importancia de la Taxonomía Clásica para la Ciencia Moderna.

Palabras clave: Región Amazónica; Arecaceae; Distribución geográfica; Urucuri.

\section{Introdução}

Arecaceae Bercht. \& J. Presl (Berchtold \& Presl, 1820) foi validamente publicado a partir de uma diagnose feita em tcheco pelos botânicos Bedřich Všemír von Berchtold e Jan Svatopluk Presl em 1820, e é uma das oito (8) famílias que apresenta o nome conservado, Palmae (Jussieu, 1789), de acordo com o artigo 18.5. do Código de Internacional de Nomenclatura para Algas Fungos e Plantas (Turland et al., 2018).

Pertencente à Ordem Arecales Bromhead (Bromhead, 1840), as Arecaceae, de acordo com The Angiosperm Phylogeny Group [APG IV] (2016), está subdividida em cinco subfamílias: Arecoideae Burnett (Burnett, 1835a), Calamoideae Beilschm. (Beilschmied, 1833), Coryphoideae Burnett (Burnett, 1835b), Ceroxyloideae Drude (Drude, 1877) e Nypoideae Griff. (Griffith, 1850).

A família das palmeiras forma um dos principais troncos da evolução das monocotiledôneas, sendo constituída por 188 gêneros e cerca de 2600 espécies (Baker \& Dransfield, 2016; Henderson et al., 1995; Palmweb, 2021). No Brasil ocorrem 37 gêneros e cerca de 300 espécies (Soares et al., 2020).

A sistemática do grupo é consensualmente baseada em características morfológicas dos estipes, das folhas, dos frutos, das flores, nas particularidades anatômicas dos seus órgãos, em conjunto a estudos de comparação de características citológicas e histológicas, distribuições geográficas e história evolutiva da família e seus gêneros (Baker \& Dransfield, 2016; Dransfield et al., 2008; Henderson, 2020; Henderson et al., 1995).

O atual sistema de classificação das plantas (APG IV, 2016), baseado em informações filogenéticas, promoveu o reagrupamento de muitas famílias botânicas e um novo arranjo na sistemática de plantas. Notoriamente, os sistemas de classificação das plantas aprimoram-se e como consequência nomes atribuídos aos táxons modificaram-se em uma nova configuração.

Extraordinariamente, na literatura científica encontram-se publicações que ao mencionarem a nomenclatura botânica de uma espécie não atentaram para a utilização de sinônimos ao invés do nome aceito, causando uma anacronia entre o 
conhecimento gerado e a relação com o objeto em estudo, que necessariamente precisa ser designado, independente da natureza da pesquisa, mas que depende do conhecimento e do engajamento dos autores e revisores.

Depreende-se que esse prospecto se deve, em parte, da dificuldade na identificação das espécies, ao desconhecimento das fontes a serem consultadas e dos procedimentos a serem adotados, pela ausência de consulta a especialistas para a determinação e elucidação dos nomes aceitos e da não linearização temporal das atualizações nas bases de dados online, que são utilizadas para consulta dos nomes válidos, distribuição geográfica e outras informações de famílias, gêneros e espécies, e que cujas informações nem sempre encontram-se em consenso único sobre determinado táxon.

Contudo, a revisão de especialistas e o acréscimo de novas informações advindas de coletas e coleções botânicas destacam o preponderante papel da Taxonomia Clássica na identificação das espécies e colaboração com as mais diversas áreas do saber. O presente estudo pretendeu discutir a complexidade taxonômica e a ampliação da área de ocorrência, para o Estado do Amapá da espécie Attalea phalerata Mart. ex Spreng. (Sprengel, 1825), popularmente denominada urucuri.

\section{Metodologia}

Esta pesquisa caracteriza-se como de natureza Taxonômica (Lawrence, 1973), cujo delineamento envolveu trabalho de campo, com coleta de amostras botânicas e revisão Taxonômica da Família, Gênero e Espécie.

A coleta foi realizada no município de Mazagão, Campus Experimental da Embrapa Amapá, em 14 de agosto de 2020

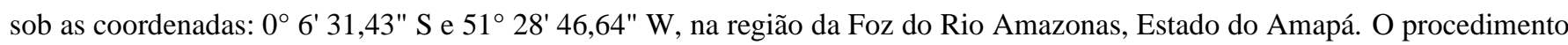
de descrição e morfometria, foi realizado no Herbário Amapaense (HAMAB) albergado no Instituto de Pesquisas Científicas e Tecnológicas do Estado do Amapá (IEPA), as fotografias e métricas foram realizadas mediante observação a olho nu. A prancha foi composta utilizando-se o software Adobe Photoshop (Knoll \& Knoll, 2020) com fotografias cedidas por Lira Guedes.

A análise morfométrica foi realizada in vivo, por meio de medidas máximas e mínimas dos caracteres florais e vegetativos com o auxílio de fita métrica. A nomenclatura morfológica baseou-se em Dransfield et al. (2008), Henderson (2020) e Radford et al. (1974). As abreviações usadas na descrição do material tipo foram: ca. = a cerca de, aproximadamente; $\mathrm{cm}$ = centímetros; compr. = comprimento; larg. = largura; e diam. = diâmetro. $\mathrm{E}$ foi utilizado o sinal de (!) para designação do tipo físico.

A escrita dos nomes genéricos foi realizada de acordo com Farr e Zijlstra (2021) contido no Index Nominum Genericorum Plantarum e para os nomes supragenéricos (família, subfamília, tribo e subtribo) foram confirmados em Reveal (2021) registrados em Indices Nominum Supragenericorum Plantarum Vascularium.

\section{Resultados}

Arecaceae Bercht. \& J. Presl, Přir. Rostlin: 266. Jan-Apr 1820, nom. alt. Areca catechu L. Lectótipo: Pinanga in Rumphius, Herb. Amboin., 1: 26, t. 4, 1741.

Arecoideae Burnett, Outlines Bot.: 401. Feb 1835.

Attalea Kunth in Humboldt, Bonpland et Kunth, Nova Gen. Sp. 1: ed. fol. 248; ed. qu. 309. Aug (sero) 1815 [1816].

Attalea phalerata Mart. ex Spreng., Syst. Veg., Ed. 16 [Sprengel] 2: 624 (1825). Neótipo: Glassman 1977b: Martius 1845: t. 169. Epítipo: Brasil. Goiás: Munícipio de Goiânia, 14 km nordeste da cidade, na Rod. BR-060/BR153 para Anápolis, 16²0'S 49¹6’W, 700-800 m, 7 maio, 1992, L. Noblick \& H. Ferreira 4864 (UFG!). Isoepítipo (FTG! NY!), conforme Figura 1. 
Figura 1 - Hábito (a) e detalhes da infrutescência (b, c, f), da inflorescência (e), da bráctea peduncular (d) e do fruto em corte transversal (g) de Attalea phalerata, Amapá, Brasil, 2021.
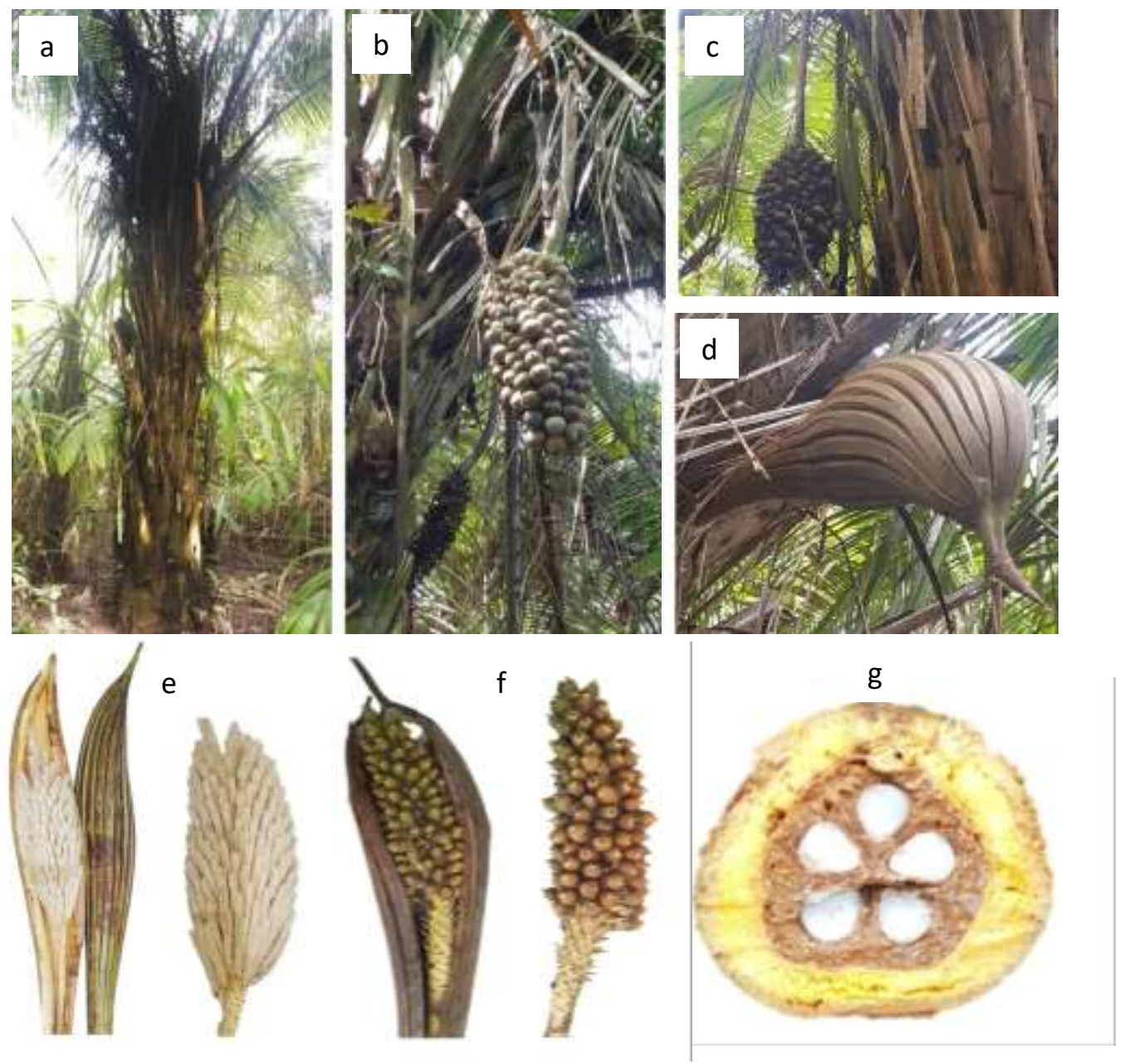

Fonte: Fotografias Lira Guedes (2021).

Descrição: Palmeira caulescente ou acaulescente, solitária ca. $8 \mathrm{~m}$ de alt. Estipe ca. $43 \mathrm{~cm}$ de diam., coberto pela base das bainhas foliares. Folhas pinadas ca. $4 \mathrm{~m}$ de compr. Bainha ca. $55 \mathrm{~cm}$ de compr. Pecíolo ca. 37-42 cm de compr. e 5-7 cm de largura na base. Ráquis ca. 3-5 m de compr., com 110-130 pares de pinas em cada lado da ráquis, com pontas acuminadas, assimétricas, arranjado irregular em diferentes planos, com aurículas nas bases. Inflorescência interfoliar unissexuada, ramificada em primeira ordem; ráquilas estaminadas 246 (110-348), 9,0 (6,0-15,0) cm de comprimento, dispostas ao redor da ráquis, com ráfides abundantes. Flores estaminadas com 7,2 (6,0-9,4) mm de comprimento, superficiais na ráquila, frouxamente e arranjados irregularmente; pétalas estaminadas lineares, livres, não curvas no ápice, superfícies estriadas, não coriáceas, não tomentosas granulares, margens lisas; estames 6, muito mais curtos do que as pétalas; anteras retas. Bráctea peduncular ca. $45 \mathrm{~cm}$ de compr., rígida, sulcada, com ápice acuminado. Pedúnculo $110 \mathrm{~cm}$ compr.; raque $80 \mathrm{~cm}$ compr., ramificada ao nível de primeira ordem. Frutos oblongo-elipsoides; epicarpo esverdeado a acastanhado; endocarpo rígido com feixes inconspícuos. Endosperma regular, 5 lóculos.

Nomes Vernaculares: acuri, bacuri, paxiba, pirinã, uricuri e urucuri (Lima \& Almeida Junior, 2020; Lorenzi et al., 2010).

Comentários: Após revisões e análises, este estudo confirma A. phalerata como nova ocorrência para o Estado do Amapá. 
Material Examinado: BRASIL, AMAPÁ: Mazagão, Campo Experimental da Embrapa Amapá, 06'31,43"S, 51²8'46,64"W, 14.VIII.2020, A.C. Lira-Guedes 01 (HAMAB).

Material Adicional Examinado: BRASIL, ACRE: Reserva Extrativista do Alto Juruá, right bank of Rio Juruá, Seringal S. João, Colocação Tapaúna, 19.III.1992, E.J. Ferreira 169 (NY); Xapurí, Seringal Cachoeira, 35 km south-south-east of Xapuri, Colocação Altamira, off verador through capoeira/campo east after 1st bridge and 1st gate, s.d., M. Pinard 858 (NY); AMAZONAS: Rodovia Manaus-Itacoatiara, km 75-70, mata alta da terra firme, 17.X.1963, E. Oliveira 2714 (IAN); GOIÁS: Cezarina, beira de estrada, ca. $200 \mathrm{~km}$ do Rio dos Bois, área de cultivo de hortaliças, 07.IX.2009, R.C. Martins 1039 (UB); MATO GROSSO DO SUL: Corumbá, Base de Estudos do Pantanal, 20.IV.2009, W.S. Fava 24772 (UB); Rio Pequiri, about 150 km S of Rondopolis, along BR-163, 01.IX.1976, S.F. Glassman, 13092 (NY); PARÁ: Barcarena, Praia do Caripí, rain forest remnants in alluvial sandy soils near the river, 18.X.1994, L.R. Noblick 5018 (IAN, NY); SÃO PAULO: Buritizal, Fazenda Caçula, acesso pelo lado esquerdo da Rodovia Buritizal-Pedregulho, km 8, estrada em direção ao fundo do vale, do lado esquerdo, a ca. de 25-50 m da bifurcação, indo para fazenda Pary, s.d., H.Q.B. Fernandes 3202 (NY).

Sinônimos: Attalea excelsa Mart. ex Spreng., Syst. Veg., Ed. 16 [Sprengel] 2: 624 (1825); Maximiliana princeps Mart., Voy. Amér. Mér. 8: t. 4 1842; Attalea princeps Mart. Voy. Amér. Mér. 7(3): 113 1844; Scheelea princeps (Mart.) H. Karst. Linnaea 28(3): 269 (1857); Scheelea amylacea Barb.Rodr., Pl. Jard. Rio de Janeiro 1: 17 1891; Scheelea leandroana Barb. Rodr. Pl. Jard. Rio de Janeiro 1: 19 1891; Scheelea anizitziana Barb.Rodr., Palm. Mattogross. 63 1898; Scheelea princeps var. corumbaensis Barb.Rodr. Palm. Mattogross. Nov. 66 (t. 21, fig. a) (1898); Scheelea quadrisperma Barb.Rodr. Palm. Paraguay 23 (t. 6) (1899); Scheelea lauromuelleriana Barb.Rodr. Contr. Jard. Bot. Rio de Janeiro 4: 108 1901; Attalea parviflora Barb.Rodr., Bull. Herb. Boissier II, 3: 625 1903; Scheelea corumbaensis (Barb.Rodr.) Barb.Rodr., Sert. Palm. Brasil. 1: 54 1903; Scheelea quadrisulcata Barb.Rodr. Contr. Jard. Bot. Rio de Janeiro 4: 107 (t. 22B) (1907); Attalea hoehnei Burret, Notizbl. Bot. Gart. Berlin-Dahlem 10: 522 1929; Scheelea gomphococca Burret Notizbl. Bot. Gart. Berlin-Dahlem 10: 541 1929; Scheelea huebneri Burret, Notizbl. Bot. Gart. Berlin-Dahlem 10: 663 1929; Scheelea martiana Burret, Notizbl. Bot. Gart. Berlin-Dahlem 10: 541 1929; Scheelea phalerata (Mart. ex Spreng.) Burret, Notizbl. Bot. Gart. Berlin-Dahlem 10: 541 1929; Scheelea tetrasticha (Drude) Burret Notizbl. Bot. Gart. Berlin-Dahlem 10: 667, 701 (1929); Scheelea weberbaueri Burret. Notizbl. Bot. Gart. Berlin-Dahlem 10: 659 (1929); Scheelea microspadix Burret, Notizbl. Bot. Gart. Berlin-Dahlem 15: 104 1940; Attalea amylacea (Barb.Rodr.) Zona, Palms (1999+) 46(3): 132 (2002); Attalea anizitsiana (Barb.Rodr.) Zona, Palms 46: 132 2002; Attalea huebneri (Burret) Zona, Palms 46: 133 2002; Attalea lauromuelleriana (Barb.Rodr.) Zona, Palms 46: 133 2002; Attalea phalerata var. concinna L.R.Moreno \& O.I.Moreno. Revista Soc. Boliv. Bot. 4: 68 2003; Attalea pacensis M.Moraes \& Pintaud, Palms 60(4): 161-168. 2016.

Distribuição Geográfica: A. phalerata distribui-se desde, Bolívia, Colômbia, Paraguai, Peru e Brasil: Acre, Amapá (aqui localizado), Amazonas, Distrito Federal, Goiás, Maranhão, Mato Grosso do Sul, Mato Grosso, Minas Gerais, Pará, Rondônia, São Paulo e Tocantins (Henderson, 2020; Govaerts et al., 2021; Soares et al., 2020; Spicieslink, 2021; Tropicos, 2021).

Status de Conservação: As informações apontam que este táxon é considerado como menos preocupante (LC), devido a sua ampla distribuição geográfica no Brasil de acordo com Centro Nacional de Conservação da Flora [CNCFLORA] (2012). Entretanto, a espécie foi considerada "Vulnerável" (VU) na Lista vermelha da flora de São Paulo segundo a Secretaria de Estado do Meio Ambiente de São Paulo [SMA-SP] (2004). E é cultivada no Jardim Botânico Plantarum (Instituto Plantarum, 2011) e no Sítio Burle Marx (Specieslink, 2021). 


\section{Discussão}

Nas florestas tropicais a Subfamília Arecoideae, Tribo Cocoseae Mart. ex Dumort. (Dumortier, 1829), se destaca por seus representantes ocorrerem em diferentes formações vegetais, cujas espécies agregam valor sociocultural e econômico para as comunidades locais.

Esta tribo compreende 17 gêneros, que foram agrupados neste clado porque apresentam inflorescência associada a um único profilo, bráctea peduncular lenhosa, gineceu, geralmente, tri-ovulado e endocarpo duro e espesso (Pintaud et al., 2008; Noblick, Hahn \& Griffith, 2013; Meerow et al., 2015; Lima \& Almeida Junior, 2020). As flores estaminadas foram usados anteriormente para segregar os gêneros, mas esse não é mais um critério aceito (Dransfield et al., 2008; Freitas et al., 2016).

Dentre os gêneros, destaca-se Attalea Kunth, que compõe a subtribo Attaleinae Drude (Drude, 1877) [Cocoseae: Arecoideae]. A Etimologia do gênero Attalea homenageia Attalus III Philometor, Rei de Pérgamo na Ásia Menor, 138-133 a.C., que posteriormente se interessou pelas plantas medicinais (Glassman, 1999; Henderson, 2020).

O gênero Attalea tem sido objeto de revisões com número de espécies aceitas variável em decorrência, principalmente, dos limites genéricos e do número de espécies adotados na taxonomia e sistemática do grupo (Freitas et al., 2016; Henderson, 2020; Zona, 2002). Para exemplificar, Glassman (1999), considerou 66 espécies para quatro gêneros: Attalea, Scheelea H.Karst., Orbignya Mart. ex Endl. e Maximiliana Mart., enquanto Henderson et al. (1995) reconheceram 29.

Várias pesquisas têm incluído os gêneros citados em Attalea, argumentando que os gêneros anteriormente reconhecidos não têm consistência (Baker \& Dransfield, 2016; Henderson, 1995; Henderson et al., 1995; Henderson, 2020).

Em revisão filogenética e biogeográfica de Attalea Freitas et al. (2016) concluíram que os grupos anteriormente reconhecidos não são monofiléticos e, portanto, não há suporte para vários gêneros como se considerava anteriormente. Mais recentemente, em revisão taxonômica do gênero com base em material de herbários e adotando o conceito de espécie filogenética, Henderson (2020) analisou 902 espécimes considerando 54 variáveis quantitativas e qualitativas passando a reconhecer 30 espécies. No World Checklist of Selected Plant Families [WCSP] (2021) o gênero possui 70 espécies. Evidenciando a discrepância dos dados gerados nas publicações das pesquisas e a disponibilização dessas informações nos indexadores, que são utilizados como fontes de consulta online.

No Brasil ocorrem 32 espécies, sendo 20 endêmicas e 28 sinônimos (Soares et al., 2020). Das quais, ressalta-se Attalea phalerata, pela importância ecológica e socioeconômica. A maioria das palmeiras nativas da Amazônia possui algum tipo de utilidade para os habitantes da região e que se converte em alguma forma de rentabilidade (Balick \& Beck, 1990; Campos \& Ehringhaus, 2003). No Amapá, essa espécie já foi objeto de estudo sobre o seu potencial produtivo dos frutos, a fim de determinar a disponibilidade de estoque para manejo e conservação e já está presente no mercado consumidor amapaense na forma de velas de uricuri (Dantas et al., 2014).

A espécie foi descrita por Karl Friedrich Philipp von Martius em trabalho assinado por Kurt Polycarp Joachim Sprengel em 1825. Já circunscrição do gênero Attalea Kunth foi realizada pelo botânico alemão Karl Sigismund Kunth (Kunth, 1816).

Morfologicamente, A. phalerata é considerada uma espécie polimórfica quanto ao estipe e pecíolo, ambos podem ser ausentes ou bem desenvolvidos (Soares et al., 2020). Consequentemente, como aqui registrado, vários sinônimos são atribuídos à espécie, além de A. excelsa Mart. (Henderson, 2020; Soares et al., 2020; Tropicos, 2021; WCSP, 2021).

Especificamente há muita semelhança morfológica entre as espécies de Attalea, um exemplo é Attalea princeps Mart. (Martius, 1844) e A. phalerata. Nota-se que ambas espécies têm causado dificuldade nas identificações. A. princeps se diferencia principalmente pelo arranjo sempre helicoidal das flores estaminadas na ráquila e o endocarpo contém densos e largos feixes de fibras dispostas de forma circular. Em A. phalerata as flores estaminadas são dispostas ao longo da face abaxial da ráquila ou raramente dispersas de forma helicoidal e o endocarpo, contém pequenos feixes de fibras dispostos de 
forma circular (Soares et al., 2020). E mais recentemente, Henderson (2020) sinonimizou A. princeps em A. phalerata (Quadro $1)$.

Quadro 1 - Comparação entre as espécies Attalea phalerata, A. princeps e A. excelsa e os sinônimos reconhecidos em bases indexadoras e Instituições de Pesquisa em Sistemática e Taxonomia de Plantas, 2021

\begin{tabular}{|c|c|c|c|c|}
\hline \multirow{2}{*}{ Indexador / Instituiçãa } & \multicolumn{3}{|c|}{ Status do Nome Científico } & \multirow{2}{*}{ Notas } \\
\hline & A. phalerata & A.princeps & A. excelsa & \\
\hline $\begin{array}{c}\text { U.S. National Plant Germplasm System (GRIN- } \\
\text { Global) / United States Department of Agriculture } \\
\text { [Usda] (2021) }\end{array}$ & Aceito & Sinônimo & Sinônimo & $\begin{array}{l}\text { Glassman (1977) abordou o } \\
\text { problema de designação do tipo }\end{array}$ \\
\hline $\begin{array}{c}\text { Flora do Brasil } 2020 \text { / Jardim Botânico do Rio de } \\
\text { Janeiro (Flora do Brasil, 2020) }\end{array}$ & Aceito & Aceito & Sinônimo & $\begin{array}{l}\text { Henderson (2020) sinonimizou } \\
\text { A. princeps em A. phalerata }\end{array}$ \\
\hline $\begin{array}{c}\text { Palm Web (Palms of the World Online) / } \\
\text { International Palm Society; Royal Botanic Gardens } \\
\text { (Kew), Botanischer Garten e Botanisches Museum } \\
\text { (Berlin-Dahlem) e Aarhus University (Palmweb, } \\
\text { 2021) }\end{array}$ & Aceito & Aceito & Sinônimo & 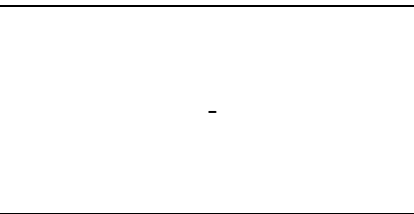 \\
\hline $\begin{array}{c}\text { Tropicos.org / Missouri Botanical Garden } \\
\text { (Tropicos, 2021) }\end{array}$ & Aceito & Sinônimo & Sinônimo & - \\
\hline $\begin{array}{l}\text { World Checklist of Selected Plant Families (WCSP) } \\
\text { / Royal Botanic Gardens (Kew Science) (WCSP, } \\
\text { 2021) }\end{array}$ & Aceito & Aceito & Sinônimo & - \\
\hline
\end{tabular}

Fonte: Autores (2021).

Não obstante, para as espécies as modificações na denominação dos nomes genéricos e epítetos específicos são processos comuns e recorrentes na sistemática vegetal e sempre adotadas as recomendações do Código Internacional de Nomenclatura para Algas, Fungos e Plantas (Bicudo et al., 2018; Turland et al., 2018), uma vez que a definição de uma espécie depende das informações verificadas nas amostras botânicas coletadas e da acurácia dos especialistas em suas interpretações que ao longo do tempo podem ser corroboradas ou não.

Portanto, muitas identificações são revalidadas e/ou retificadas, de acordo com o conhecimento que se dispõe, do trabalho dos taxonomistas, de novas coletas, das informações de Herbários e do status de preservação dos tipos nomenclaturais.

\section{Considerações Finais}

O gênero Attalea corresponde a um grupo bastante complexo, principalmente pela variação morfológica, e que ao longo do tempo tem passado por revisões que recircunscreveram várias espécies resultando em nomes de espécies consideradas aceitas e muitas sinonimizações.

A partir da realização desta pesquisa amplia-se a distribuição geográfica de Attalea phalerata para o Estado do Amapá. Confirmando a importância de estudos florístico-taxonômicos no contexto do conhecimento e valorização da flora brasileira.

Conclui-se que a importância da taxonomia vegetal se justifica, dentre vários aspectos, também no sentido de esclarecer e sustentar o emprego das denominações específicas e genéricas aceitas, reforçando a importância da Taxonomia Clássica para a Ciência Moderna. 


\section{Referências}

Balick, M. J., \& Beck, H. T. (1990). Useful palms of the world: A synoptic bibliography. Columbia University.

Baker, W. J., \& Dransfield, J. (2016). Beyond Genera Palmarum: Progress and prospects in palm systematics. Botanical Journal of the Linnean Society, 182, 207-233. https://academic.oup.com/botlinnean/article/182/2/207/2707815.

Barbosa Rodrigues, J. (1891). Plantas Novas Cultivadas no Jardim Botânico do Rio de Janeiro, descriptas, classificadas e desenhadas por J. Barbosa Rodrigues Director do mesmo jardim I. https://upload.wikimedia.org/wikipedia/commons/b/b0/Plantas_novas_cultivadas_no _Jardim_Bot\%C3\%A2nico_do_Rio_de_Janeiro.pdf.

Barbosa Rodrigues, J. (1898). Palmae Mattogrossenses Novae Vel Minus Cognitae quas collegit descripsit et iconibus iIlustravit J. Barbosa Rodrigues, Eques Antiqui, Nobitissimi, atque Clarissimi Ordinis Saneti Jacobi a Gladio, Director Horti Bataeiei Fluminis Januarii, Socius Effectivus Instituti Historiei et Gcographici Braziliae, Laureatus ab Instituto Scientiarum Physicarum et Naturalium Florentiae, Socius Regiae Academiae Scientiarum Olysipponensis, Imperialis et Regalis Societatis Botanicae Vindobonensi, Societatum Botanicae Anedae et Massiliae, Instituti Conimbricensis, Regiae Societatis Anthropologicae Florentiae, Societatis Friburgucosis Investigatorum Naturac, Natiooalis Academiae Parisiorum, neenoo Societatis Geograpbicae Parisiorum, et Fluminis Januarii, etc. Rio de Janeiro Typographia Leuzinger. https://www2.senado.leg.br/bdsf/handle/id/242527.

Barbosa Rodrigues, J. (1899). Palmae Novae Paraguayenses quas descripsit et iconibus illustravit J. Barbosa Rodrigues Director Hortus Fluminensis, Typographia Leuzinger. https://www2.senado.leg.br/bdsf/item/id/242528.

Barbosa Rodrigues, J. (1901). Ordo Palmae Mart. Contributions du Jardin Botanique de Rio de Janeiro par son Directeus J. Barbosa Rodrigues I. Typographie LEtoile du Sud, Rua de S. José 102. https://ia600402.us.archive.org/9/items/contributionsduj14jard/contributionsduj14jard.pdf.

Barbosa Rodrigues, J. (1903a). Sertum Palmarum Brasiliensium ou relation des palmiers nouveaux du Brésil découverts, décrits et dessinés par, d'apres nature J. Barbosa Rodrigues, Directeur du Jardin botanique de Rio de Janeiro, professeur de botanique de l'Université libre de Rio-de-Janeiro, chevalier du tres noble et tres ancien Ordre de Saint-Jacques et de 1'Ordre royal de la Couronne d'Italie, membre de l'Institut historique, géographique et ethnologique du Brésil, de l'Institut polytechnique brésilien, lauréat de l'Institut des sciences physiques et naturelles de Florence, membre de l'Académie royale des sciences de Lisbonne, de la Société impériale et royale de botanique de Vienne, de la Société foyale de botanique de Bruxelles, de la Société de botanique de Marseille, de la'Société de botanique d'Edimbouro, de la ociété royale anthropologique et ethnologiqlle de Florence, de la Société des Naturalistes de Freiburg, de l'Institut de Coimbra, des Sociétés géographique de Rio-de-Janeiro et de Paris, 'vice-président honoraire du dellxieme Congres scientifique latin-américain, etc. Première Partie. Imprimerie Typographique Veuve Monnom, Bruxeles. 32, Rue de L'industrie, 32. https://www2.senado.leg.br/bdsf/handle/id/242529.

Barbosa Rodrigues, J. (1903b). Sertum Palmarum Brasiliensium ou relation des palmiers nouveaux du Brésil découverts, décrits et dessinés par, d'apres nature J. Barbosa Rodrigues, Directeur du Jardin botanique de Rio de Janeiro, professeur de botanique de l'Université libre de Rio-de-Janeiro, chevalier du tres noble et tres ancien Ordre de Saint-Jacques et de 1'Ordre royal de la Couronne d'Italie, membre de l'Institut historique, géographique et ethnologique du Brésil, de l'Institut polytechnique brésilien, lauréat de l'Institut des sciences physiques et naturelles de Florence, membre de l'Académie royale des sciences de Lisbonne, de la Société impériale et royale de botanique de Vienne, de la Société foyale de botanique de Bruxelles, de la Société de botanique de Marseille, de la'Société de botanique d'Edimbouro, de la ociété royale anthropologique et ethnologiqlle de Florence, de la Société des Naturalistes de Freiburg, de l'Institut de Coimbra, des Sociétés géographique de Rio-de-Janeiro et de Paris, 'vice-président honoraire du dellxieme Congres scientifique latin-américain, etc. Seconde Partie. Imprimerie Typographique Veuve Monnom, Bruxeles. 32, Rue de L'industrie, 32. https://www2.senado.leg.br/bdsf/handle/id/242529.

Barbosa Rodrigues, J. (1903c). Palmae det. J. Barbosa Rodriguez. Mémoires de l'Herbier Boissier suite au bulletin de l'Herbier Boissier. https://www.biodiversitylibrary.org/item/105266\#page/668/mode/1up.

Barbosa Rodrigues, J. (1907). Suplementarum ad Sertum Palmarum Brasiliensium. Contributions du Jardin Botanique de Rio de Janeiro par son Directeus J. Barbosa Rodrigues IV. Typographie LEtoile du Sud, Rua de S. José 102. https://ia600402.us.archive.org/9/items/contributions duj14jard/contributionsduj14jard.pdf.

Beilschmied, C. T. (1833). Schottische Gebirgs - Vegetation am Fluße Dee in Aberdeenshire. Nach W. Macgillivray. Aus Mem. of the Wern Soc. VI. (Edinb. 1832.) im Auszuge mitgetheilt. In: Flora oder Allgemeine Botanische Zeitung, 16: 641-656. https://www.zobodat.at/publikation_volumes.php?id=61202.

Berchtold, B. V., \& Presl, J. S. (1820). O Prirozenost Rostlin obratugici gednánj o ziwobytj rostlin pro sebe az ohledu giných žitoků podle stawu nyněgss ho znánj pýtwu rostlin názwoslomj audi̊ hospodárstwj gegic rozsfjčenj po zemi a způsob rostlinář zřjditi a zachowati Wydáno trudem Berchtold Bedřicha Wssemjra Hrabéte z Berchtoldu a Sana Swatopluka Presla Doktorůw lekarstwoj Pra er Bollani o farla Wilima Enderra toiltupce to malé jezuité aliele. https://books.google.com.br/books?redir_esc=y\&hl=pt-BR\&id=M3oQAQAAMAAJ\&q=areca\#v=onepage \&q=areca\&f=true.

Bicudo, C. E. M., Prado, J., \& Hirai, R. Y. (2018). Código Internacional de Nomenclatura Botânica para algas, fungos e plantas (Código de Shenzhen, 2018). RIMA.

Bromhead, E. T. F. (1840). Remarks on the Botanical System of Professor Perleb. By Sir Ewd. Ff. Bromhead, Bt. F.R.S., Lond. And Ed. In: Charlesworth, E. The Magazine of natural history, n.s. 4, 333. https://www.biodiversitylibrary.org/item/19629\#page/356/mode/1up.

Burnett, G. T. (1835a). Outlines of Botany, including a General History of the Vegetable Kingdom, in which Plants are arrenged according to the System of Natural Affinities, By Gilbert T. Burnett, F.L.S. Professor of Botany in King's College, London and Senior President of the Westminster Medical Society. v. I.

Burnett, G. T. (1835b). Outlines of botany, including a general history of the vegetable kingdom, in which plants are arranged according to the system of natural affinities. By Gilbert T. Burnett, f.l.s. Professor of Botany in King's College, London; and Senior President of the Westminster medical Society. vol. I. London: John Churchill, Princes Street, Soho. MDCCCXXXV. https://archive.org/details/b28742631_0001/page/n5/mode/2up.

Burret, M. K. E. (1929). Die Palmengattungen Orbignya, Attalea, Scheelea und Maximiliana. Notizblatt des Botanischen Gartens und Museums zu BerlinDahlem 10, na 1. https://www.jstor.org/stable/3994776?refreqid=excelsior\%3Ad8127f70937fb77701fa351828b24160\&seq=1

Burret, M. K. E. (1940). Palmen von A. C. Smith aus Brit. Guyaana. Notizblatt Des Königl. Botanischen Gartens Und Museums Zu Berlin-Dahlem, 15(1). http://www.jstor.org/stable/3995085. 
Campos, M. T., \& Ehringhaus, C. (2003). Plant virtues are in the eyes of the beholders: a comparison of known palm uses among indigenous and folk communities of southwestern amazonia. Economic Botany, 57(3), 324-344.

Centro Nacional de Conservação da Flora. (2012). Attalea phalerata in Lista Vermelha da flora brasileira versão 2012.2. http://cncflora.jbrj.gov.br/portal/ptbr/profile/Attaleaphalerata.

Cook, O. F. (1910). Contributions from The United States National Herbarium, 13, part. 5 Relationships of the Ivory Palms. https://ia800607.us.archive.org/7/items/mobot39088011696762/mobot39088011696762.pdf.

Cronquist, A. (1988). The evolution and classification of flowering plants. 2. ed. The New York Botanical Garden.

Dantas, A. R., Ribeiro, G. G., Lira Guedes, A. C., \& Guedes, M. C. (2014). Produção de frutos de urucurizeiros, Attalea excelsa Mart. (Arecaceae), em floresta de várzea no estuário do rio Amazonas. Biota Amazônia, 4(4), 107-113. https://periodicos.unifap.br/index.php/biota/article/view/1210.

Dransfield, J., Uhl, N. W., Asmussen, C. B., Baker, W. J., Haley, M. M., \& Lewis, C. E. (2008). Genera Palmarum: The evolution and classification of palms. Kew Publishing, Royal Botanical Gardens, Kew, UK. https://kew.iro.bl.uk/concern/books/503db94e-b77d-4f40-8707-1d1b602b2b86?locale=en.

Drude, C. G. O. (1877). Ausgewählt Beispiele zur Erläuterung der Fruchtbildung bei den Palmen. Botanische Zeitung, 39. https://www.biodiversity library.org/item/104882\#page/354/mode/1up.

Drude, C. G. O. (1822). Palmae. Vol. III, Part II, Fasc. 86 Coluna 579. In: Martius C. F. V. Flora Brasiliensis. http://florabrasiliensis.cria.o rg.br/search?taxon_id=1639.

Dumortier, B. (1829). Analyse des familles des plantes: avec l'indication des principaux genres qui s'y rattachent. Tournay. https://www.biodiversitylibrary.org/item/32871\#page/3/mode/1up.

Farr, E. R., \& Zijlstra, G. (2021). Index Nominum Genericorum (Plantarum). Atualizado continuamente. https://naturalhistory2.si.edu/botany/ing/.

Freitas, C., Meerow, A. W., Pintaud, J.-C., Henderson, A., Noblick, L., Costa, F. R. C., Barbosa, C. E., \& Barrington, D. (2016). Phylogenetic analysis of Attalea (Arecaceae): Insights into the historical biogeography of a recently diversified neotropical plant group. Botanical Journal of the Linnean Society, 128, 287-302.

Glassman, S. (1977). Preliminary taxonomic studies in the palm genus Attalea H.B.K. Fieldiana Botany, 38, 31-61. https://doi.org/10.5962/bhl.title.2446.

Glassman, S. (1999). A taxonomic treatment of the palm subtribe Atttaleinae (Tribe Cocoeae). Illinois Biological Monographs, 59, 1-414. https://doi.org/10.5962/bhl.title.49920.

Govaerts, R., Dransfield, J., Zona, S., Hodel, D. R., \& Henderson, A. (2021). World Checklist of Arecaceae. Facilitated by the Royal Botanic Gardens, Kew. Published on the Internet. http://wcsp.science.kew.org/Retrieved.

Griffith, W. (1850). Posthumous Papers bequeated to the honourable the East India Company, and printed by order of the Government of Bengal. Palms of British East India, by the late William Griffith, ESQ. F.L.S. https://gallica.bnf.fr/ark:/12148/bpt6k98250v.image\#.

Henderson, A. (1995). Palms of the Amazon. Oxford University Press.

Henderson, H., Galeano, G., \& Bernal, R. (1995). Field guide to the palms of the Americans. Princeton University Press.

Henderson, A. (2020). A revision of Attalea (Arecaceae, Arecoideae, Cocoseae, Attaleinae). Phytotaxa, 444(1), 1-76. https://doi.org/10.11646/phytotaxa.444.1.1.

Instituto Plantarum. (2011). Acervo Vivo: Jardim Botânico Plantarum. http://www.plantarum.org.br/jardim-botanico.

Jussieu, A. L. (1789). Genera Plantarum: secundum ordines naturales disposita, juxta methodum in Horto regio parisiensi exaratam, anno M.DCC.LXXIV. https://www.biodiversitylibrary.org/bibliography/284\#/summary.

Karsten, G. K. W. H. (1856). Plantae Columbianae. Linnaea: Ein Journal für die Botanik in ihrem ganzen Umfange, 28(12). https://www.biodiversitylibrary.org/item/10872\#page/272/mode/1up.

Knoll, T., \& Knoll, J. (2020). Adobe Photoshop. Adobe Systems. www.adobe.com/products/photoshop.html.

Kunth, K. S. (1816). Nova genera et species plantarum: quas in peregrinatione ad plagam aequinoctialem orbis novi collegerunt /descripserunt, partim adumbraverunt Amat. Bonpland et Alex. de Humboldt ; ex schedis autographis Amati Bonplandi in ordinem digessit Carol. Sigismund. Kunth. Accedunt Tabulae Aeri Incisae, et Alexandri de Humboldt Notationes Ad Geographiam Plantarum Spectantes. Tomus Primus. Lutetiae Parisiorum, Suntibus Librariae Graeco-Latino-Germanicae, Via Dicta Fossés-Montmartre, 14. 1815. https://www.biodiversitylibrary.org/item/270966\#page/9/mode/1up.

Lawrence, G. H. M. (1973). Taxonomia das plantas vasculares. 1. v. Fundação Calouste Gulbenkian.

Le Maout, E., \& Decaisne, J. (1868). Traité de Botanique Descriptive et Analytique. https://books.googleusercontent.com/books/content ?req=AKW5Qacxzw39e loFZE_JzLMiLirVwh-8jVKuU1IajUrcGG0C1pU6gAoZ-2JRS9Bwcd-m3hqB7wdUgVS8BGui_C1EYzrnyg9VOFUdLGF 84cQG2 wm mmfVL2Xck96XJ1Q8QFwaMsYGVd1MKo6F10ybR-jHTYry_pdq9LOnU2kh9neLp0Z36Q8hJZHDxsw5gPII5vGVZzTOGtK0-q3v086c0SEY12 M6px _OGbmIJYZwb2mH8k7wfevNp40cbP83Cxv7y-XPWaPRHqL7OfzfLITZMM-X-0MdW6ST4EwHoyZcztsGcP6YDNBzNJs.

Lima, G. P., \& Almeida Junior, E. B. (2020). Synopsis of the tribe Cocoseae Mart. (Arecoideae, Arecaceae) in the state of Maranhão, Brazil. Biota Neotropica, 20(2), e20190922. https://www.scielo.br/j/bn/a/cNfqJ6WcrwB6HrsC8rn5txH/?lang=en.

Lorenzi, H., Noblick, L., Kahn F., \& Ferreira, E. (2010). Flora Brasileira: Arecaceae (palmeiras). Instituto Plantarum. 
Martius, C. F. V. (1844). Voyage dans l'Amerique Meridionale (Le Brésil, La République Orientale De L'Uruguay, La République Argentina, La Patagonia, La République du Chili, La République de Bolivia, La République de Pérou), exécuté pendant les années 1826, 1827, 1828, 1829, 1830, 1831, 1832 et 1833, par Alcide D'Orbigny, chevalier de l'ordre royal de la legion d'honneur, officier de la Legion D'honneur bolivienne, Vice-président de la Societe Geologique de France et membre de plusieurs Academies et Societes Savantes Nationales et Etrangeres. Ouvrage dédie au roi, et publié sous le áuspices de M. le Ministre de L'Instruction publique (commence sous le ministère de M. Guizot). Tome Troisième. $3^{\text {re }}$. partie. Paris, Chez P. Bertrand, Éditeur, Libraire de la Société Géologique de France, Rue Saint-André-Des-Arcs, 38. Strasbourg, Chez V.e Levrault, Rue Des Juifs, 33, 1834-1847. https://ia800204.us.archive.org/4/items/voyagedanslamriq00orbi_1/voyagedanslamriq00orbi_1.pdf.

Meerow, A. W., Noblick, L., Salas Leiva, D. E., Sanchez, V., Francisco Ortega, J., Jestrow, B., \& Nakamura, K. (2015). Phylogeny and historical biogeography of the cocosoid palms (Arecaceae, Arecoideae, Cocoseae) inferred from sequences of six WRKY gene family loci. Cladistics, $31(5), 509-534$. https://doi.org/10.1111/cla.12100.

Moraes, M. R., \& Pintaud, J. (2016). A new species of Attalea from Bolivian lowlands. Palms, 60(4), 161-168. https://www.researchgate.net/publication/311910369_A_new_species_of_Attalea_from_Bolivian_lowlands/figures.

Moreno, L. R., \& Moreno, O. I. (2003). Revista de la Sociedad Boliviana de Botánica, 4(1), 68. http://resbbo.sbb.org.bo/publicaciones/

Noblick, L. R., Hahn, W. J., \& Griffith, M. P. (2013). Structural cladistic study of Cocoseae, subtribe Attaleinae (Arecaceae): evaluating taxonomic limits in Attaleinae and the neotropical genus Syagrus. Brittonia, 65(2), 232-261. https://www.jstor.org/stable/24692576.

Palmweb. (2021). Palmweb: palms of the world online. http://www.palmweb.org.

Perleb, C. J. (1838). Clavis Classiüm, Ordinum Et Familiarum Atqub Index Generüm Regni Vegetabilis. Diagnostische Uebersichtstafeln Des natürlichen Pflanzensystems. Nebst vollständigem Gattungsregister. Von Dr. C. J. PERLEB, ord. öffentl. Professor der Naturgeschichte und Botanik an der grossh. bad. lluüfertï̈̈ts Froiburg, üirector de» akademücken Nuturaliencahineltee u. hotan. Gartens, mehr. gel. Gen. Milgl Freibürg im Br., bei Adolph Emmerling. https://archive.org/details/clavisclassiumor00perl.

Pintaud, J.-C., Galeano, G., Balslev, H., Bernal, R., Borchsenius, F., Ferreira, E., Granville, J.-J., Mejía, K., Millán, B., Moraes, M., Noblick, L., Stauffer, F. W., \& Kahn, F. (2008). Las palmeras de América del Sur: diversidad, distribución e historia evolutiva. Rev. Peru. Biol., Suplemento 1, 15, 7-29. https://dialnet.unirioja.es/descarga/articulo/2874711.pdf.

Radford, A. E., Dickison, W. C., Massey, J. R., \& Bell, C. R. (1974). Vascular plant systematics. Harper \& How Publishers.

Reveal, J. L. (2021). Indices Nominum Supragenericorum Plantarum Vascularium: alphabetical listing by genera of validly published supragenericnames. http://www.plantsystematics.org/reveal/pbio/fam/allspgnames.html.

Schultz, K. H. S. (1832). Natürliches System des Pflanzenreichs nach seiner inneren Organisation. Nebst einer vergleichenden Darstellung der wichtigsten aller früheren künstlichen und natürlichen Pflanzensysteme. Hirschwald, https://books.google.com.br/books?id=xw0AAAAAQAAJ\&pg=PA35\&lpg=PA35\&dq=Nat\%C3\%BCrliches+System+des+Pflanzenreichs+nach+seiner+inner en+Organisation\&source=bl\&ots=cUG5gN774u\&sig=ACfU3U36caNeiy-CDLbVO7zVq34kkxgzDA\&hl=pt-

$\mathrm{BR \& sa}=X \&$ ved=2ahUKEwit58q7vpzvAhW5HbkGHTLLBpI4ChDoATASegQIDxAD\#v=onepage\&q=Nat\%C3\%BCrliches\%20System\%20des\%20Pflanzenr eichs\%20nach\%20seiner\%20inneren\%20Organisation.

Secretaria de Estado do Meio Ambiente de São Paulo. (2004). Resolução SMA N. 48 de 2004. Lista oficial das espécies da flora do Estado de São Paulo ameaçadas de extinção, Diário Oficial do Estado de São Paulo, São Paulo, SP. https://www.infraestruturameioambiente.sp.gov.br/institutodebotanica/wpcontent/uploads/sites/235/2014/02/resolu\%C3\%A7\%C3\%A3o_-sma48.pdf.

Soares, K. P., Lorenzi, H., Vianna, S. A., Leitman, P. M., Heiden, G., Moraes, R. M., Martins, R. C., Campos Rocha, A., Ellert Pereira, P. E., \& Eslabão, M. P. (2020). Arecaceae in Flora do Brasil 2020. 2020. Jardim Botânico do Rio de Janeiro. http://floradobrasil.jbrj.gov.br/reflora/floradobrasil/FB53.

Soares, K. P., Longhi, S. J., Witeck Neto, L., \& Assis, L. C. (2014). Palmeiras (Arecaceae) no Rio Grande do Sul, Brasil. Rodriguésia, 65(1), 113-139. http://www.scielo.br/scielo.php?script=sci_arttext\&pid=S2175-78602014000100009\&lng=en\&nrm=iso.

Specieslink. (2021). Herbário da Embrapa Recursos Genéticos e Biotecnologia (CEN), Herbário CPAP da Embrapa Pantanal (CPAP), Herbário Amapaense (HAMAB), Herbário do Instituto de Estudos Costeiros da Universidade Federal do Pará (HBRA), Herbário da Universidade Estadual de Feira de Santana (HUEFS), Herbário do Instituto Agronômico de Campinas (IAC), Herbário INPA (INPA), Missouri Botanical Garden - Brazilian records (MOBOT_BR), Smithsonian Department of Botany - Brazilian records (NMNH-Botany_BR), The New York Botanical Garden - Brazilian records (NY), Herbário do Museu Nacional (R), Herbário do Estado "Maria Eneyda P. Kaufmann Fidalgo" - Coleção de Fanerógamas (SP), Herbário UFP - Geraldo Mariz (UFP). http://www.splink.org.br.

Sprengel, C. (1825). Equitis stellar polaris, archiatri regii, prof. Rei herb. in Univers. Upsal. Systema vegetabilium. Editio decima sexta, curante, Curtio Sprengel, Equite stellae polaris rubrae, Prof. Med. et rei Herb. In Univers. Hal. Volumen II. Calssis 6-15. Gottingae, Sumtibus librariae dieterichianae. https://www.biodiversitylibrary.org/page/2922624\#page/1/mode/1up.

The Angiosperm Phylogeny Group. (2016). An update of the Angiosperm Phylogeny Group classification for the orders and families of flowering plants: APG IV. Botanical Journal of the Linnean Society, 181, 1-20. https://academic.oup.com/botlinnean/article/181/1/1/2416499.

Tropicos. (2021). Tropicos.org. Missouri Botanical Garden. http://www.tropicos.org.

Turland, N. J., Wiersema, J. H., Barrie, F. R., Greuter, W., Hawksworth, D. L., Herendeen, P. S., Knapp, S., Kusber, W. H., Li, D. Z., Marhold, K., May, T. W., Mcneill, J., Monro, A. M., Prado, J., Price, M. J., \& Smith, G. F. (2018). International Code of Nomenclatura for algae, fungi, and plants (Shenzhen Code) adopted by the Nineteenth International Botanical Congress Shenzhen, China, July, 2017. Regnum Vegetabile 159. Glasshütten: Koeltz Botanical Books. https://www.iapt-taxon.org/nomen/main.php.

United States Department of Agriculture. (2021). Agricultural Research Service, National Plant Germplasm System. Germplasm Resources Information Network (GRIN Taxonomy). National Germplasm Resources Laboratory, Beltsville, Maryland. https://npgsweb.arsgrin.gov/gringlobal/taxon/taxonomygenus?id=1150. 
Research, Society and Development, v. 10, n. 11, e119101119294, 2021

(CC BY 4.0) | ISSN 2525-3409 | DOI: http://dx.doi.org/10.33448/rsd-v10i11.19294

World Checklist of Selected Plant Families. (2021). Attalea. https://wcsp.science.kew.org/qsearch.do.

Zona, S. (2002). Name changes in Attalea. Palms, The Journal of the The International Palm Society, 46(3). https://palms.org/resources/palms-journal/\#. 